Vegan toxicology:

In vitro methods offer an alternative to animal

testing for drug safety

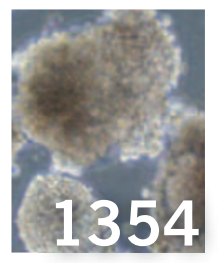

Cellular potential:

Stem cell biologists

look for a new way to

measure pluripotency

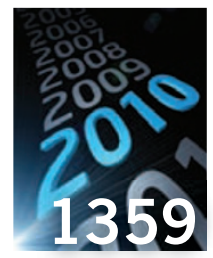

Year in review:

Our annual look back

at the year that was

in biomedicine

\title{
Newer antidepressants go beyond serotonin-and the synapse
}

In the US, many of the nearly one in ten people suffering from depression rely on so-called 'third generation' antidepressants, which typically alter the reuptake of the signaling molecule serotonin. It's no secret, though, that these drugs can take weeks to kick in, and sometimes carry serious side effects.

However, in the past few months alone, researchers have discovered a suite of potential drug targets for depression. Unlike the previous targets, such as serotonin, dopamine and norepinephrine, which act at the synapse between neurons, the newer targets act within the brain cells.

"By targeting some of the intracellular mechanisms that are modulated by classical antidepressants in a very slow way, maybe we can achieve faster therapeutic responses," says Olivier Berton, a neuroscientist at the University of Pennsylvania in Philadelphia.

Last month, for instance, Yale University researcher Ronald Duman and his colleagues reported in this journal that an enzyme called mitogen-activated protein kinase phosphatase-1 was abundant in postmortem brain tissue taken from people with depression. Altering the expression of this protein in mice and rats affected the animals' depressive behaviors and stress responses, the researchers found (Nat. Med. 16, 1328-1332, 2010).

In August, Duman and his coworkers also reported that ketamine, a fast-acting antidepressant with a previously unknown mode of action, works by activating a specific signaling pathway_involving the 'mammalian target of rapamycin', or mTOR-to increase the number of connections between neurons in the executive region of the brain (Science 329, 959-964, 2010). The insight opens up the possibility of developing other $\mathrm{mTOR}$ activators as treatments for depression, which is key because ketamine can cause hallucinations. (For more on this idea, see 'Lifting the mood with ketamine' on page 1384.)

Meanwhile, an enzyme previously implicated in several neurodegenerative diseases, cyclindependent division protein kinase-5 (Cdk5), also seems to have a role in depression. At the Society for Neuroscience's annual meeting in San Diego last month, James Bibb of the

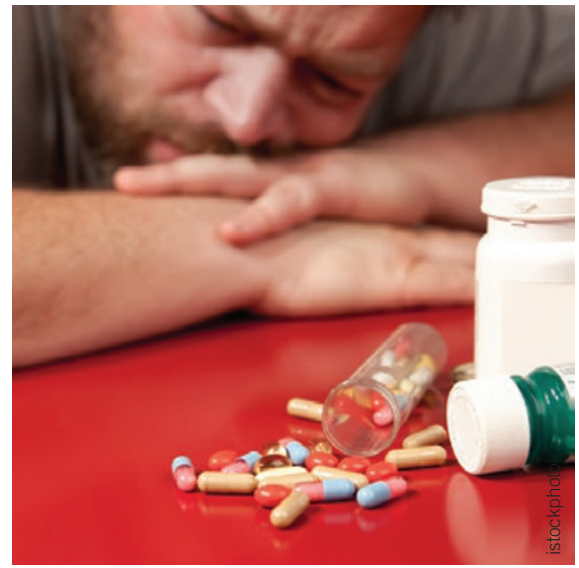

Up on antidowners: New depression targets.

University of Texas Southwestern Medical Center in Dallas reported that mice engineered to lack the enzyme showed the same protection against stress as mice given antidepressants. Cdk5 seems to regulate essential intracellular signaling molecules. "This represents an important pathway that could be targeted to improve moods and remove people from overwhelming states of despair," Bibb says.

\section{Old dog, new tricks}

Researchers have not completely abandoned the idea of treating depression by manipulating the action of signaling molecules. For example, a team led by Fang Liu at the Centre for Addiction and Mental Health in Toronto are targeting the dopamine pathway. On page 1393 of this issue of Nature Medicine, Liu's team reports that postmortem brain samples from people with major depression showed that the dopamine cell receptors D1 and D2 clump together. Treating rats with a peptide that disrupted this coupling produced significant antidepressant-like effects, the researchers found.

By far, the pathway most commonly targeted by existing antidepressant drugs is the serotonin system. Yet, instead of affecting levels of the neurotransmitter itself, Berton has his sights set on an enzyme linked to serotonin. In a series of experiments presented at the Society for Neuroscience meeting, Berton showed that the enzyme histone deacetylase-6 (HDAC6) is rife in the serotonin-signaling neurons of depressed mice and people. What's more, knocking out HDAC6, either genetically or with broad-acting drugs, caused the mice to be more resilient in stressful situations, Berton found.

John Traynor and his colleagues at the University of Michigan-Ann Arbor have meanwhile discovered that modulating the ' $1 \mathrm{a}$ ' receptor for serotonin alone might efficiently treat depression. At present, most selective serotonin reuptake inhibitor (SSRI) antidepressants increase cell signaling by indiscriminately acting on more than 20 serotonin receptors in the brain. Reporting in June, Traynor's team showed that disabling the action of the serotonin 1a receptor caused mice to spend more time in exposed environments and act less jittery in a marble-burying testboth indications of an antidepressant effect (Proc. Natl. Acad. Sci. USA 107, 11086-11091, 2010).

"It's all about the selectivity issue," explains Traynor. "If we can find the right serotonin receptor, then a drug should have a more rapid onset, and it should be more selective with fewer side effects."

Separately, in August scientists from Paris Descartes University showed that fluoxetine (Prozac) works by increasing brain levels of a particular microRNA called miR-16 in certain types of neurons, which in turn slows the formation of the serotonin transporter protein that clears away the neurotransmitter from the brain's synapses (Science 329, 1537-1541, 2010). Thus, the researchers propose, people suffering from depression could be treated with mediators of miR-16 to experience more targeted moodlifting benefits than current drugs.

Still, Thomas Cremers, chief executive of Brains On-Line, a neurochemistry contract research company based in San Francisco, cautions that several promising approaches, including targeting the neuropeptide substance $\mathrm{P}$ and corticotrophin-releasing hormone, have failed to prove effective in clinical trials. "They all look promising, but to turn them into druggable therapies is something different," Cremers says. "We'll have to wait and see if anyone can push them into the clinic. That's the next step."

Elie Dolgin 